\title{
Proposing a framework for specifying the appropriateness of pursuing a career regarding Employees' healthiness and safeguard: A Case study of food manufacturing industry
}

\author{
Ayria Behdinian ( $\nabla$ ayria.behdinian@ut.ac.ir) \\ University of Tehran https://orcid.org/0000-0002-7772-4854 \\ Kamran Rezaie \\ University of Tehran \\ Ali Bozorgi-Amiri \\ University of Tehran
}

\section{Research}

Keywords: employees, health, human, position

Posted Date: October 13th, 2021

DOl: https://doi.org/10.21203/rs.3.rs-955123/v1

License: (c) (1) This work is licensed under a Creative Commons Attribution 4.0 International License.

Read Full License 


\section{Abstract}

\section{Background}

Employee health is an essential issue for Human Resource Management (HRM). The employees' health level is undeniably correlated to the job position in which they work since it may harm their well-being, and they may not be capable of performing their duties properly. Prompt diagnosis and resolution of employees' physical complications are highly critical.

\section{Methods}

Machine learning $(\mathrm{ML})$ is the state-of-the-art method potentially utilized to make early predictions to safeguard employees' healthiness. The technical laborers within the food manufacturing company are included in this Research. The functional classification models, namely, Support Vector Machine (SVM), K-Nearest Neighbors (KNN), Logistic Regression (LR), Decision Tree, are exploited to predict the employees' wellness for their vocation. K-fold Cross-Validation (KCV) and Confusion Matrix were applied in this study, the former for estimating the model's functionality and the latter for forecasting accuracy.

Results

After implementing four models on the 231 employees, the accuracy was extracted out, SVM with $78 \%$, KNN with $78 \%$, Decision Tree with $80 \%$, and the highest for LR algorithm with $84 \%$.

Conclusions

In this Research, the LR algorithm was opted to paving the way for Human Resources Managers in order to utilize a functional system to predict the Suitability of factory workers concerning their healthiness. The Hearing condition was picked out as a leading factor in selecting employees for their job position. Consequently, it is significant to planning a hearing conservation program for employees, especially those exposed to excessive noise.

Trial Registration: Retrospectively registered.

\section{Contributions To The Literature}

- Applied Machine Learning Algorithms to build predictive models for recognizing the Appropriateness of an employee's work condition concerning their Medical symptoms.

- Highlighted the employees' hearing condition as an underlying factor in pursuing the profession in the manufacturing industry.

- Utilized a system to decrement the time cycle and cost of staff examination regularly.

- Proposed a system to take health level into account for selection and recruiting the staff. 


\section{Introduction And Background}

One of the most valuable assets of an organization is human resources, and the organization's growth and development highly depend on planning for employees (Desarno et al., 2021). Human Resources Management area revolves around recruiting, hiring, deploying, and managing an organization's employees (Rihan, 1998).

One of the most significant issues that HR managers should take into consideration is the Health and safety of employees in the workplace (Perera, 2019; Mosadeghrad, 2013; Anger et al., 2015). There is an undeniably direct correlation between job performance and employees' health conditions (Lukas et al., 2007), especially in manufacturing firms (Perera, 2019). Taking employees' Health into consideration in the workplace by managers assists prevent possible injuries in the workplace and puts workforces in their proper positions (Linnander et al., 2017; Williams and Skinner, 2003); moreover, the job insecurity rate will be reduced if applicable employees were recruited (Jiang et al., 2021). Johnson et al. (2017) stated that physical potential for employees is one of the substantial factors for managers to employ workforces. Shaffer and Gilbert (2018) mentioned that Adherencing to health standards in the workplace leads to protecting employees from hazardous and immune families from adverse effects of harming in workspaces. Guerin et al. (2020) referred to Many work environments with special conditions, especially in the production plant environment where the risk to employees is higher; hence, it is essential to concentrate on employees' Health in such environments. Identifying diseases and physical conditions level of employees is costly for the organization, and it is laborious for the organization to repeat it regularly (Ross et al., 2016); hence managers look forward to predicting employees' health conditions by approaches that are time-saving and less costly (Wu et al., 2019) (Smiti, 2020).

Machine Learning is the state-of-the-art method that can be utilized to identify the pattern in the data and predict the outcome according to data (Motwani et al., 2017). Different types of ML algorithms can be developed to identify diseases and unhealthy employees to intervene adverse effects on workforces and companies (Liu et al., 2021). Birjais et al. (2019) mentioned ML algorithms for the diagnosis of Diabetes disease; for doing this, three algorithms were employed, named Gradient Boosting, Logistic Regression, and naïve Bayes, with the higher accuracy of $86 \%$ for the Gradient Boosting. Cui et al. (2020) utilized Random Forest as a functional ML algorithm for forecasting the major depressive disorder (MDD) with an accuracy of $73.4 \%$ among 1500 samples. The adverse effects of medical conditions on workers were measured utilizing a classification method called Logistic Regression; high cholesterol and high glucose were the two main risks for the workers' Health (Gerassis et al., 2021). Pahuja and Nagabhushan (2021) exploited ML algorithms, named Artificial neural Network (ANN), Support Vector Machine, and K-nearest neighbor for Parkinson's' disease detection, ANN opted as the best classifier with the accuracy of $95.89 \%$.

Apart from ML applications in disease diagnoses, it is employed to predict the employees' workplace health. ML algorithms are perfectly functional in the field of health management in the organization, and the improvement in the health level can be recognized (López-Martínez et al., 2020). Reddy et al. (2018) analyzed mental Health in the workplace and could highlight the effectiveness of family history as an 
underlying factor in these types of disorders by utilizing the Decision Trees algorithm. Zhao et al. (2019) attempted to predict the noise-induced hearing impairment of laborers exposed to various complex occupational noises with four classification models, which resulted that the best model for this prediction is SVM, which could gain $80.1 \%$ accuracy.

In this paper, medical factors were utilized for Human Resource Management purposes. According to the literature, it is recognizable that machine learning methods have been widely employed in disease diagnosis. However, These algorithms are less applied in predicting the Health of human resources employees in organizations, especially the manufacturing industry, where employees experience complicated conditions. After concerning medical features, exploiting the underlying ML algorithms to predict the Suitability to carry on working in factory workplace conditions for employees is an actionable decision for the HRM department.

\section{Problem Description}

Staff health assessment and determining whether the position they worked at is appropriate or may harm them is a leading factor in HRM. Managers must safeguard the workers' health conditions in the manufacturing industry to prevent side effects the workplace may have for the workers, furthermore; It is significant to recognize that each individual can work in a specific position in the factory due to their health level. Apart from this, during recruitment, a novel approach can be utilized to determine whether a person is perfectly fitted for that position or the workplace may harm one's Health. Managers look forward to exploiting the efficient, time-saving method and have reasonable accuracy in predicting appropriate individuals for the manufacturing industry according to their Healthiness level. ML algorithms can be perfectly applied to recognize the effectiveness of medical components on a career, consequently paving the way for human resources managers to select the most productive individuals. The whole process of the research problem and solving is displayed in Fig 1.

\subsection{Case Study}

The case utilized in this Research is a food manufacturing factory. The employees who worked at the production line participated in this paper. Blood tests and general practitioners' examinations were the basis for forming factors involved in this study. This center's employees' tasks revolve mainly around packaging, labeling, or storing food for human consumption and providing food for sale or distribution to other business entities such as food processing plants or food establishments. This factory and the many food chain industries have poor conditions in terms of considering laborers' Health; however, hazards in this environment, especially facing ear-splitting facilities, should be considered so as not to affect employees' Health.

\subsection{Factors}

Labors' general Health has multiple factors which can be evaluated to recognize the physical Health of workers. For implementing the model, it is necessary to take the features into account which is related to 
the professional environment and can affect employees' task implementation (Chaudoir et al., 2013; Milat et al., 2015). Collection and preparation of information related to blood and urine can be utilized as a factor in assessing individuals' health conditions (Cornelis et al., 1996). Blood composition is plasma, red blood cells, white blood cells, and platelets, all of which can be normal, high, or low in the medical examination measurement; thus, according to the amount of these factors in the blood, health level can be assessed (Sun et al., 2019). Urine is another factor constituting Electrolytes, nitrogenous chemicals, vitamins, hormones, organic acids, which should be considered for healthiness (Karak and Bhattacharyya, 2011). Many tasks are performed in the close viewing distance in the workplace condition; thus, to carry out responsibility with high potential, employees' eye vision within the factory is critical to be examined (Anshel, 2006). Hearing conditions and their protection are substantially significant in the workplace, especially in laboratory conditions; hence, employees' hearing levels have to be determined (Nélisse et al., 2012). Audiometry test consists of five levels: Normal (less than 25 DB HL), Mild (25-50 DBHL), Moderate (41-65 DB HL), Severe (66-90 DB HL), Profound (more than $90 \mathrm{DBHL}$ ) (Davies, 2016) and Notch (hearing loss at 3 to $6 \mathrm{kHz}$ ) (Nélisse et al., 2012). The Standard threshold for hearing has to be considered in the workplaces, and appropriate employees have to be set for positions according to their hearing level (Daniell et al., 2003). Age and gender are two other factors that affect the work environment and employee performance (Wilks and Neto, 2013). In this paper, all of the factors mentioned above have been utilized to present a functional healthcare system for employees to determine whether pursuing their career is appropriate or may harm their physical condition. The criteria for measuring the factors are summarized in Table 1.

Table1. The Factors

\begin{tabular}{|c|c|c|}
\hline Factors & Type & Range \\
\hline \multirow[t]{2}{*}{ Gender } & Categorical & Male $=1$ \\
\hline & & Female $=0$ \\
\hline Age & Numeric & $21-64$ \\
\hline \multirow[t]{3}{*}{ Blood and Urine } & Categorical & Normal \\
\hline & & High $\left(\mathrm{RBC}^{\star}, \mathrm{Hb}^{\star}, \mathrm{MCHC}^{\star}, \mathrm{PLT}^{\star}, \mathrm{MCV}^{\star}, \mathrm{MCH}^{\star}, \mathrm{HCT}^{\star}, \mathrm{WBS}^{\star}\right)$ \\
\hline & & Low ((FBS*,RBC,Hb,MCHC,PLT,MCV,MCH,HCT,WBS) \\
\hline Left eye vision & Numeric & $1-10$ \\
\hline Right eye vision & Numeric & $1-10$ \\
\hline Left ear hearing condition & Categorical & Normal-Mild-Moderate-Severe-Profound-Notch \\
\hline Right ear hearing condition & Categorical & Normal-Mild-Moderate-Severe-Profound-Notch \\
\hline \multirow[t]{2}{*}{ Pursuing job } & Categorical & Yes $=1$ \\
\hline & & $\mathrm{No}=0$ \\
\hline
\end{tabular}


*Red blood cells, ${ }^{*}$ Hemoglobin, ${ }^{*}$ Mean cell hemoglobin concentration,*Platelets, ${ }^{*}$ Mean corpuscular volume,*Mean corpuscular volume, ${ }^{*}$ Hematocrit, ${ }^{\star}$ White blood cell,*Fasting blood sugar

\section{Methodology}

\subsection{Machine Learning}

The main goal of this study was to propose a system to predict whether a job position is appropriate for a specific worker or may harm physical health. For this purpose, after processing raw data (data transformation and normalization) to be suitable for utilizing ML algorithms, four classification models, namely, Logistic Regression, Support Vector Machine, Decision Tree, K-Nearest-Neighbors, were fitted on the dataset. Among these algorithms, LR is proposed for this study since it has the highest accuracy. LR is a functional approach in classification models, widely employed in assessing data in sociology, biostatistics, clinical medicine, quantitative psychology, econometrics, marketing, and many other areas (Dreiseitl and Ohno-Machado, 2002).

For the implementation of the LR Algorithm, the best parameter for the $b_{0}$ and $b_{1}$ is to be picked out. The equation for LR is as follows (Hosmer et al., 2000):

$$
y=\frac{e^{\left(b_{0}+b_{1} * X\right)}}{1-e^{\left(b_{0}+b_{1} * X\right)}}
$$

Here $y$ is the predicted output, and $b_{0}$ (the bias or intercept term) and $b_{1}$ (the coefficient for the single input value $x$ ) are to be chosen by minimizing the LR cost function. The equation for LR cost function is as follows:

$$
J(\theta)=-\frac{1}{m} \sum_{i=1}^{m}\left[y^{(i)} \log \left(h_{\theta}\left(X^{(i)}\right)\right)+\left(1-y^{(i)}\right) \log \left(1-h_{\theta}\left(X^{(i)}\right)\right)\right]
$$

\subsubsection{Cross-validation}

Many algorithms can be employed to assess the ML models, namely, Train/test split, $k$-fold CrossValidation (CV), Leave-one-out Cross-Validation (LOOCV), Leave-one-group-out Cross-Validation (LOGOCV), Nested Cross-Validation (NCV), Wilcoxon signed-rank test, Mc Nemar's test, etc. The k-fold cross-validation is one of the most practical techniques for estimating the classifier's error (Anguita et al., 2012). The KCV process consists of splitting a dataset into k subsets; afterward, iteratively, some of them are utilized to build the model, while the others are exploited to analyze its performance.

\subsection{Model Validation}


The Confusion Matrix is a widely used method to recognize the correlation between the actual and predicted values (Xu et al., 2020). The Precision, Recall, F1-score, and Accuracy metrics are calculated based on the Confusion Matrix. The formulas for these metrics are as follows (Townsend, 1971):

$$
\begin{gathered}
\text { Precision }=\frac{T P}{T P+F P} \\
\text { Recall }=\frac{T P}{T P+F N} \\
\text { Accuracy }=\frac{T P+T N}{T P+T N+F P+F N} \\
F 1-\text { score }=\frac{T P}{T P+\frac{1}{2}(F P+F N)}
\end{gathered}
$$

TP, TN, FP, and FN are defined as follows:

- True Positive (TP): The outcome where the model correctly predicts the positive class.

- True Negative (TN): The outcome where the model correctly predicts the negative class.

- False Positive (FP): The outcome where the model incorrectly predicts the positive class.

- False Negative (FN): The outcome where the model incorrectly predicts the negative class.

\section{Numerical Results}

\subsection{Feature Importance}

After Transforming Data structures to Standard form, it is significant to recognize the correlation between independent variables and dependent variables. Heatmap Visualization is a functional method for simultaneously representing the correlation between all variables (Pryke et al., 2007). The 2-D Heat Map for this Research has been represented in Fig. 2.

It is conceivable that the most contributing feature for pursuing a job is a Normal hearing condition; therefore, a healthy hearing condition has to be considered during recruitment and picking an individual for a specific career position in the factory. After highlighting the factor importance, The ML algorithms were implemented.

\subsection{Machine Learning Results}

As the dataset is small, employing a functional technique called Cross-Validation to evaluate the classifier to prevent overfitting is significant (Moreno-Torres et al., 2012). For this purpose, the five-fold 
Cross-Validation technique is utilized in this Research. Since the LR algorithm has the highest accuracy, the confusion Matrix for LR is demonstrated in Fig. 3.

Based on the Confusion Matrix, the Precision, Recall, F1-Score, and Accuracy can be extracted. In Table 2, the metrics mentioned above for all applied algorithms are represented.

Table 2

Summary of four Classification models with 5 fold cross-Validation

\begin{tabular}{|lllll|}
\hline \multirow{2}{*}{ Algorithm } & \multicolumn{3}{l}{$\mathbf{5}$ fold Cross-Validation } & \\
\cline { 2 - 5 } & Precision (\%) & Recall (\%) & F1-score (\%) & Accuracy (\%) \\
\hline Logistic Regression & 84 & 84 & 84 & 84 \\
\hline Decision Tree & 79 & 80 & 78 & 80 \\
\hline SVM & 79 & 78 & 77 & 78 \\
\hline KNN & 79 & 78 & 76 & 78 \\
\hline
\end{tabular}

According to the results, The most practical algorithms to predict whether a job position is appropriate for an employee or may harm one's physical condition is the LR model with an accuracy of $84 \%$.

The metrics, as mentioned earlier, are also calculated for each class, and the outcome is presented in Table 3. The numerical result reports that the proposed model is better performed at predicting the yes labels in the model.

Table 3

Classification report

\begin{tabular}{|llll|}
\hline Class & Metrics & & \\
\cline { 2 - 4 } & Precision (\%) & Recall (\%) & F1-score (\%) \\
\hline 0 & 75 & 71 & 73 \\
\hline 1 & 88 & 90 & 89 \\
\hline \multicolumn{2}{|l}{ Declarations section } & \\
\hline
\end{tabular}

\section{Discussion}

The results marked that the LR algorithm with relatively high accuracy is highly functional in predicting whether working at that job position is unimpeded or the current situation is not suitable concerning one's health level. In addition, forecasting assists employees in avoiding the physical damage that they are prone to in the factory environment. The magnitude of this problem is determined; for instance, the hearing loss rate for workers exposed to occupational noise in the textile factory is $24 \%$ higher than those who are not exposed to the noise (Shakhatreh et al., 2000). According to the Importance of this issue, 
researchers predict the possibility of hearing loss in noisy workplaces (Aliabadi et al., 2015). Hence, providing high-quality healthcare in a factory environment should be considered (Mosadeghrad, 2014; Bradley et al., 2009). Workers exposed to excessive noise in the workplace have to control the noise by utilizing hearing protection, and in some cases, this issue is highly significant that the employer has to implement a hearing conservation program for workers (Sriopas et al., 2016).

After making a prediction, human resources should be directed to address the problem if they have a health problem or take steps to protect them from further harm. In any case, for employees who are not in a suitable position, the possibility of pursuing a job has to be considered, including sending them to a specialist to fix the problem, or utilizing protective equipment, or performing outpatient treatment by the general practitioners who have performed the examinations.

\section{Managerial Insights}

When considering a managerial perspective, the time and budget spent by the supervisors to implement a method are significant. In this paper, the proposed system assists the human resources manager determine the Suitability of industrial workers for their occupation, which prevents potential injuries to employees and boosts employee performance. The system provides the situation for performing these operations in less time with High accuracy; As a result of implementing this method, the organization's cost will be reduced due to diminishing employees' injuries and health risks.

The critical point in human resource management is to hire efficient employees for higher productivity. This procedure can also check employees' Health at hiring and be introduced as a contributing technique in the recruitment process. The level of health of employees is considered less during hiring; therefore, it has a practical utilization for the organization and human resource managers.

\section{Limitations}

In this paper, some limitations affected the whole process. First, the captured data was from a food manufacturing industry, and the number of persons who participated in this Research was only 231 members; the dataset was formed according to examination by a general practitioner. Second, Concerning the small dataset, the possibility of facing overfitting is high; therefore, five-fold crossvalidation was utilized to prevent overfitting. Third, a broad number of features can be applied for recognizing the health level; however, eight independent factors were utilized to develop the Machine Learning Models in this Research since the available dataset was restricted to these factors.

\section{Conclusion}

In this Research, Machine Learning Algorithms, named SVM, KNN, LR, and Decision Tree, were utilized for training captured data to predict the feasibility of pursuing a career for employees concerning their healthiness. Fivefold cross-validation was employed for the model evaluation, resulted in opting LR 
algorithm as the most contributing method; furthermore, The confusion matrix represented the $84 \%$ accuracy for the model mentioned above. Eight independent features were applied to predict the dependent variable in the classification model, named pursuing a job with yes and no labels. The 2D Heat Map displayed the amount of correlation between variables; Hearing condition was the leading feature for workers to pursue their jobs in a factory workplace. According to this Research, relying on advancement in computer Science (ML algorithms) can assist managers to take physical condition into account for opting employees for specific career positions.

For future Research, Taking advantage of other health factors to determine the suitability of the job for employees can be considered. Apart from the physical problems of the body, which can be solved mainly through applying protective facilities and referring to specialists, considering mental Health can be another vital factor, which should be taken into consideration for managers. Moreover, employing many other novel ML algorithms may lead to better performance in this area, which researchers can utilize to train on the captured data.

\section{Declarations}

\section{Ethics approval and consent to participate:}

Not applicable.

\section{Consent for publication:}

Not applicable.

\section{Availability of data and materials:}

The dataset during the current study is available from the corresponding author on reasonable request.

\section{Competing interests:}

The authors declare that they have no competing interests.

\section{Funding:}

Not applicable.

\section{Authors' contributions:}

Ayria Behdinian prepared materials, data collection, data analysis, developed the Models and drafted the manuscript. Kamran Rezaie and Ali Bozorgi-Amiri commented on and aided in the drafting of the manuscript. All authors read and approved the final manuscript.

\section{Acknowledgments:}


Not applicable.

\section{References}

1. Aliabadi M, Farhadian M, Darvishi E. Prediction of hearing loss among the noise-exposed workers in a steel factory using artificial intelligence approach. Int Arch Occup Environ Health. 2015;88(6):77987.

2. Anger WK, Elliot DL, Bodner T, et al. Effectiveness of total worker health interventions. J Occup Health Psychol. 2015;20(2):226.

3. Anguita D, Ghelardoni L, Ghio A, et al. (2012) The 'K'in K-fold cross validation. 20th European Symposium on Artificial Neural Networks, Computational Intelligence and Machine Learning (ESANN). i6doc. com publ, 441-446.

4. Anshel JR. Visual ergonomics in the workplace. Professional Safety. 2006;51(8):20.

5. Birjais R, Mourya AK, Chauhan R, et al. Prediction and diagnosis of future diabetes risk: a machine learning approach. SN Applied Sciences. 2019;1(9):1-8.

6. Bradley EH, Curry LA, Ramanadhan S, et al. Research in action: using positive deviance to improve quality of health care. Implementation science. 2009;4(1):1-11.

7. Chaudoir SR, Dugan AG, Barr CH. Measuring factors affecting implementation of health innovations: a systematic review of structural, organizational, provider, patient, and innovation level measures. Implementation science. 2013;8(1):1-20.

8. Cornelis R, Heinzow B, Herber R, et al. Sample collection guidelines for trace elements in blood and urine. J Trace Elem Med Biol. 1996;10(2):103-27.

9. Cui L, Wang C, Wu Z, et al. Symptomatology differences of major depression in psychiatric versus general hospitals: A machine learning approach. J Affect Disord. 2020;260:349-60.

10. Daniell WE, Stover BD, Takaro TK. Comparison of criteria for significant threshold shift in workplace hearing conservation programs. Journal of occupational environmental medicine. 2003;45(3):295304.

11. Davies R. Audiometry and other hearing tests. Handb Clin Neurol. 2016;137:157-76.

12. Desarno J, Perez M, Rivas R, et al. Succession Planning Within the Health Care Organization:: Human Resources Management and Human Capital Management Considerations. Nurse Leader. 2021;19(4):411-5.

13. Dreiseitl S, Ohno-Machado L. Logistic regression and artificial neural network classification models: a methodology review. J Biomed Inform. 2002;35(5-6):352-9.

14. Gerassis $S$, Boente $C$, Albuquerque $M$, et al. Mapping occupational health risk factors in the primary sector-A novel supervised machine learning and Area-to-Point Poisson kriging approach. Spatial Statistics. 2021;42:100434.

15. Guerin RJ, Castillo D, Hendricks KJ, et al. Preparing the future workforce for safe and healthy employment. American Public Health Association; 2020. 
16. Hosmer DW, Lemeshow S, Sturdivant RX. (2000) Applied logistic regression. Wiley New York.

17. Jiang $L, X u X$, Wang H-J. A resources-demands approach to sources of job insecurity: A multilevel meta-analytic investigation. J Occup Health Psychol. 2021;26(2):108.

18. Johnson CY, Rocheleau CM, Lawson CC, et al. (2017) Factors affecting workforce participation and healthy worker biases in US women and men. Ann Epidemiol 27(9): 558-62. e552.

19. Karak T, Bhattacharyya P. Human urine as a source of alternative natural fertilizer in agriculture: A flight of fancy or an achievable reality. Resources conservation recycling. 2011;55(4):400-8.

20. Linnander EL, Mantopoulos JM, Allen N, et al. Professionalizing healthcare management: a descriptive case study. International Journal of Health Policy Management. 2017;6(10):555.

21. Liu Y-X, Liu X, Cen C, et al. Comparison and development of advanced machine learning tools to predict nonalcoholic fatty liver disease: An extended study. Hepatobiliary \& Pancreatic Diseases International; 2021.

22. López-Martínez F, Núñez-Valdez ER, García-Díaz V, et al. A case study for a big data and machine learning platform to improve medical decision support in population health management. Algorithms. 2020;13(4):102.

23. Lukas CV, Holmes SK, Cohen AB, et al. Transformational change in health care systems: an organizational model. Health care management review. 2007;32(4):309-20.

24. Milat AJ, Bauman A, Redman S. Narrative review of models and success factors for scaling up public health interventions. Implementation science. 2015;10(1):1-11.

25. Moreno-Torres JG, Sáez JA, Herrera F. Study on the impact of partition-induced dataset shift on \$ k \$fold cross-validation. IEEE Transactions on Neural Networks Learning Systems. 2012;23(8):1304-12.

26. Mosadeghrad AM. Quality of working life: an antecedent to employee turnover intention. International Journal of Health Policy Management. 2013;1(1):43.

27. Mosadeghrad AM. Factors influencing healthcare service quality. International Journal of Health Policy Management. 2014;3(2):77.

28. Motwani M, Dey D, Berman DS, et al. Machine learning for prediction of all-cause mortality in patients with suspected coronary artery disease: a 5-year multicentre prospective registry analysis. European heart journal. 2017;38(7):500-7.

29. Nélisse H, Gaudreau M-A, Boutin J, et al. Measurement of hearing protection devices performance in the workplace during full-shift working operations. Annals of occupational hygiene. 2012;56(2):22132.

30. Pahuja G, Nagabhushan T. A comparative study of existing machine learning approaches for Parkinson's disease detection. IETE Journal of Research. 2021;67(1):4-14.

31. Perera G. (2019) Occupational health and safety practice and job performance: Role of job satisfaction. Sri Lankan Journal of Human Resource Management 9(1).

32. Pryke A, Mostaghim S, Nazemi A. (2007) Heatmap visualization of population based multi objective algorithms. International conference on evolutionary multi-criterion optimization. Springer, 361-375. 
33. Reddy US, Thota AV, Dharun A. (2018) Machine learning techniques for stress prediction in working employees. 2018 IEEE International Conference on Computational Intelligence and Computing Research (ICCIC). IEEE, 1-4.

34. Rihan I. (1998) What is Human Resources Management. Obtido de academia. edu: https://www. academia. edu/7775792 \&\#8230.

35. Ross J, Stevenson F, Lau R, et al. Factors that influence the implementation of e-health: a systematic review of systematic reviews (an update). Implementation science. 2016;11(1):1-12.

36. Shaffer RM, Gilbert SG. Reducing occupational lead exposures: Strengthened standards for a healthy workforce. Neurotoxicology. 2018;69:181-6.

37. Shakhatreh FM, Abdul-Baqi KJ, Turk MM. Hearing loss in a textile factory. Saudi Med J. 2000;21(1):58-60.

38. Smiti A. When machine learning meets medical world: Current status and future challenges. Computer Science Review. 2020;37:100280.

39. Sriopas A, Chapman RS, Sutammasa S, et al. (2016) Occupational noise-induced hearing loss in auto part factory workers in welding units in Thailand. Journal of occupational health. 15-0291-OA.

40. Sun S, Lulla A, Sioda M, et al. Gut microbiota composition and blood pressure: the CARDIA study. Hypertension. 2019;73(5):998-1006.

41. Townsend JT. Theoretical analysis of an alphabetic confusion matrix. Perception Psychophysics. 1971;9(1):40-50.

42. Wilks DC, Neto F. Workplace well-being, gender and age: Examining the 'double jeopardy'effect. Soc Indic Res. 2013;114(3):875-90.

43. Williams ES, Skinner AC. Outcomes of physician job satisfaction: a narrative review, implications, and directions for future Research. Health care management review. 2003;28(2):119-39.

44. Wu C-C, Yeh W-C, Hsu W-D, et al. Prediction of fatty liver disease using machine learning algorithms. Comput Methods Programs Biomed. 2019;170:23-9.

45. Xu J, Zhang Y, Miao D. Three-way confusion matrix for classification: A measure driven view. Information sciences. 2020;507:772-94.

46. Zhao Y, Li J, Zhang M, et al. Machine learning models for the hearing impairment prediction in workers exposed to complex industrial noise: a pilot study. Ear hearing. 2019;40(3):690.

\section{Figures}




\section{Transforming Data}

\section{Feature importance}

\section{Model Building}

\section{Model Evaluation}

\section{Figure 1}

The problem and solving process

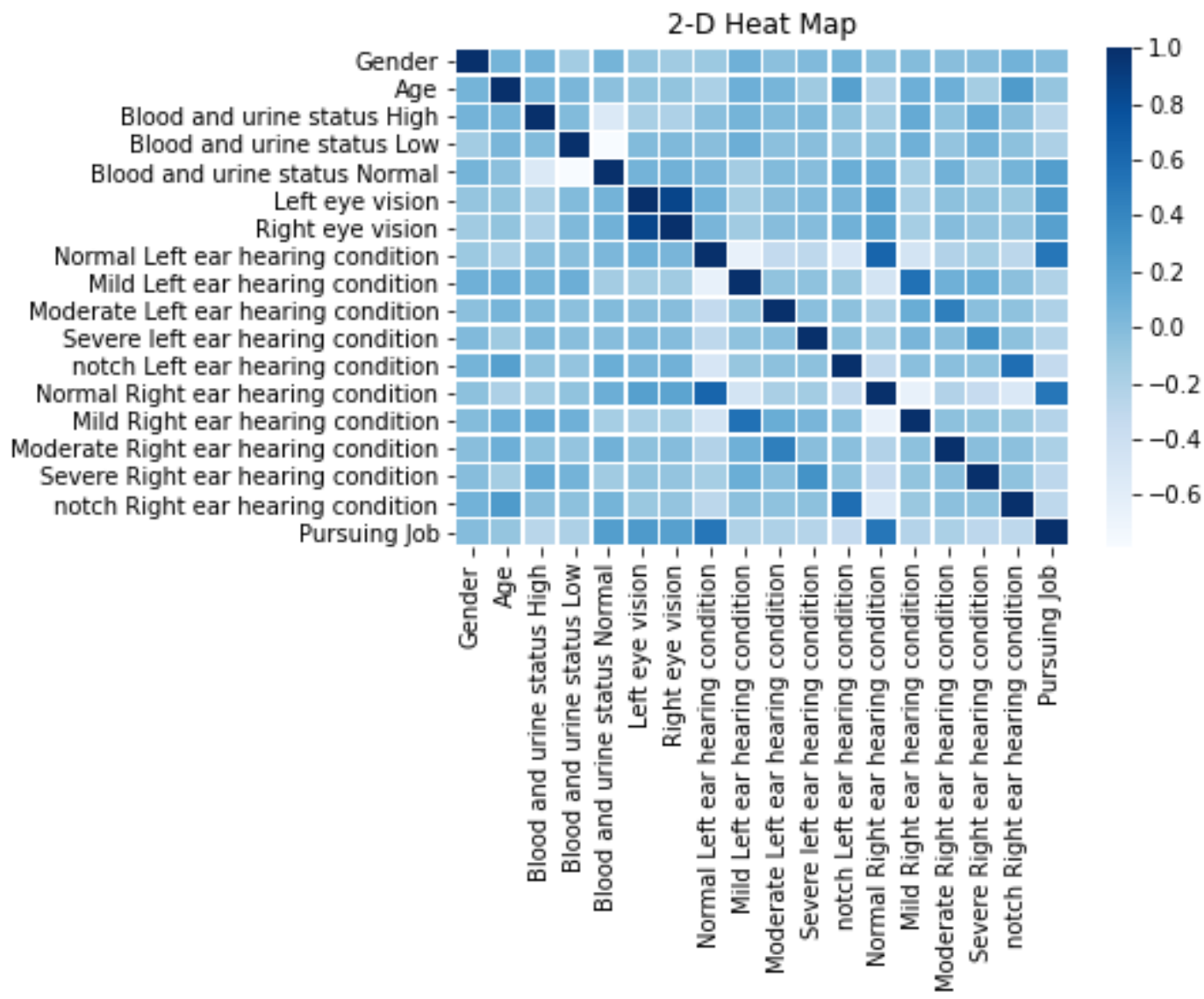

\section{Figure 2}

The problem and solving process 


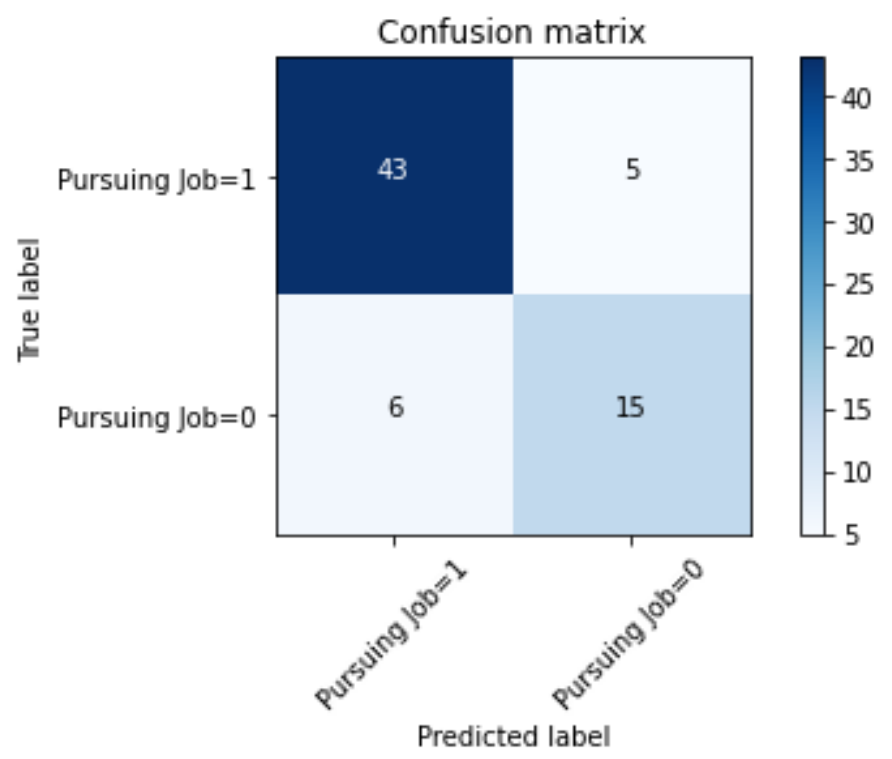

Figure 3

Confusion Matrix

\section{Supplementary Files}

This is a list of supplementary files associated with this preprint. Click to download.

- RepotingChecklist.pdf 